(c) 2016 IEEE. Personal use of this material is permitted. Permission from IEEE must be obtained for all other uses, in any current or future media, including reprinting/republishing this material for advertising or promotional purposes, creating new collective works, for resale or redistribution to servers or lists, or reuse of any copyrighted component of this work in other works 


\title{
On Existence and Stability of Equilibria of de LTI Circuits with Constant Power Loads*
}

\author{
Nikita Barabanov ${ }^{1}$, Romeo Ortega ${ }^{2}$, Robert Griñó $^{3}$ and Boris Polyak ${ }^{4}$
}

\begin{abstract}
This paper studies the problem of existence and stability of equilibria of dc linear time-invariant circuits with constant power loads. First, we correct an unfortunate mistake in our previous work [10] pertaining to the sufficiency of the condition for existence of equilibria in multiport systems given there. Second, we give two necessary conditions for existence of equiibria. The first one is a simple linear matrix inequality hence it can be easily verified with existing software. Third, we prove that the latter condition is also sufficient if a set defined by the problem data is convex, which is the case for single and two-port systems. Finally, sufficient conditions for stability and instability for a given equilibrium point are given. The results are illustrated with two benchmark examples.
\end{abstract}

\section{INTRODUCTION AND PROBLEM FORMULATION}

The ever increasing use of power electronic devices in electrical systems has given rise to a new paradigm for the representation of their dynamic loads. Indeed, due to these devices the loads do not behave as standard impedances, instead they are more accurately represented as constant power loads (CPLs), which correspond to first-third quadrant hyperbolas in the loads voltage-current plane. It has been experimentally observed that the presence of these CPLs strongly impinges on the dynamic behavior of the electrical system and may induce erratic or unstable behavior, see [10] and references therein. For this reason it is of interest to carry out a theoretical analysis of the impact of CPL on the dynamic behavior of the system and, in particular, on their ability to operate in stable steady-state.

In this paper we mainly look at linear time invariant (LTI) DC systems with CPLs which are modeled as

$$
y(t)=G(s) u(t)+k
$$

where $s$ is the Laplace variable, $G(s) \in \mathbb{R}^{m \times m}(s)$, the set of $m \times m$ rational matrices with real coefficients and

*N. Barabanov, R. Ortega and B. Polyak were supported by Government of Russian Federation (grant 074-U01, GOSZADANIE 2014/190 (project 2118)), the Ministry of Education and Science of Russian Federation (project 14.Z50.31.0031).The work of R. Griñó was partially supported by the Government of Spain through the Ministerio de Economía y Competitividad Project DPI2013-41224-P and by the Generalitat de Catalunya through the project 2014SGR 267.

${ }^{1}$ N. Barabanov is with the Department of Mathematics, North Dakota State University, Fargo, ND 58105, USA (nikita.barabanovendsu.edu).

${ }^{2} \mathrm{R}$. Ortega is with the Laboratoire des Signaux et Systèmes, Centre National de la Recherche Scientifique-École Supérieure d'Électricité (CNRS-SUPÉLEC), Gif-sur-Y vette 91192, France (ortegalis.supelec.fr).

${ }^{3} \mathrm{R}$. Griñó is with the Institute of Industrial and Control Engineering (IOC), Universitat Politècnica de Catalunya, 08028-Barcelona, Spain (roberto.grino@upc.edu).

${ }^{4} \mathrm{~B}$. Polyak is with the Institute of Control Sciences and Energy Center, SkolTech, , Moscow, Russia (boriseipu.ru). $k \in \mathbb{R}^{m}$. The port variables ${ }^{1} y, u \in \mathbb{R}^{m}$, with elements $y_{i}, u_{i}, i \in \mathcal{M}:=\{1, \ldots, m\}$, are conjugated variables, i.e., their product $y_{i} u_{i}$ has units of power. The port variables are connected to CPLs defined as

$$
-y_{i}(t) u_{i}(t)=P_{i}>0, i \in \mathcal{M},
$$

that holds for all $t \geq 0$.

We are interested in two questions.

Q1. Give conditions on the system and load parameters for the existence of constant steady-state behavior. In particular, for practical reasons, it is desirable to define the maximal power that can be extracted from the source, i.e., $\sum_{i=1}^{m} P_{i}$, ensuring the good behaviour of the system.

Q2. Assuming a steady-state behavior exists, under which conditions the associated equilibrium point is (Lyapunov) stable or unstable.

The remaining of the paper is organized as follows. Section II corrects an unfortunate mistake in [10]. Section III gives two necessary condition for existence of a steadystate, with the first expressed in terms of feasibility of a linear matrix inequality (LMI). In Section IV we discuss situations when the LMI necessary condition is also sufficient, which reduces to checking the convexity of a set defined by the problem data. The stability analysis of a given equilibrium point is carried out in Section V. Section VI presents two benchmark examples. The case of nonlinear port-Hamiltonian $(\mathrm{pH})$ systems with CPLs that, as shown in [10] includes a class of power converters, is briefly discussed in Section VII. The paper is wrapped-up with concluding remarks in Section VIII.

\section{Correction to the Claim of [10]}

In [10] we addressed the question of existence of constant steady-states for the system (1), (2), a regime which is defined as follows.

Definition 1: The system (1), connected to CPLs via (2) admits a constant steady-state if and only if there exist constant vectors $\bar{u}, \bar{y} \in \mathbb{R}^{m}$ such that

$$
\begin{aligned}
\bar{y} & =G(0) \bar{u}+k \\
\bar{y}_{i} \bar{u}_{i} & =-P_{i}, i \in \mathcal{M} .
\end{aligned}
$$

In [10] the following positive definiteness assumption is made:

$$
G(0)+G^{\top}(0)>0
$$

\footnotetext{
${ }^{1}$ In the interest of brevity, when clear from the context, the argument $t$ is omitted from the time functions.
} 
As explained in Remark 3 of [10] this is reasonable in the scenario of interest. Under this assumption, it is claimed in Proposition 1 of [10] that a necessary and sufficient condition for existence of a constant steady-state is

$$
\frac{1}{2} k^{\top}\left[G(0)+G^{\top}(0)\right]^{-1} k \geq \underline{1}_{m}^{\top} P
$$

where $\mathbf{1}_{m}:=\operatorname{col}(1, \ldots, 1) \in \mathbb{R}^{m}$ and $P:=$ $\operatorname{col}\left(P_{1}, \ldots, P_{m}\right) \in \mathbb{R}^{m}$. Unfortunately, this statement is true only for $m=1$, for $m>1$ condition (6) is necessary, but not sufficient. Indeed, in Proposition 1 of [10] the definition of existence of a constant steady-state is erroneously given as existence of constant vectors $\bar{u}, \bar{y} \in \mathbb{R}^{m}$ such that (3) and the scalar condition

$$
\bar{y}^{\top} \bar{u}=-\mathbf{1}_{m}^{\top} P,
$$

hold. Notice that (7), instead of (4), is used in the definition of steady-state. Clearly, (4) implies (7), but not the other way around.

As discussed in Remark 3 of [10], if $G(s)$ is the driving point impedance of a circuit consisting of (positive) constant resistors, inductors and capacitors, with the elements of $u$ and $y$ voltages and currents, and $k$ representing constant, external current and voltage sources, there is a clear physical interpretation of condition (6). Indeed, in this case $\frac{1}{2} k^{\top}\left[G(0)+G^{\top}(0)\right]^{-1} k$ is an upper bound on the power dissipated in steady-state, that should exceed the extracted constant power to ensure the existence of equilibria. See the example in Subsection V.A of [10].

\section{Two NECESSARy CONDITIONS FOR EXISTENCE OF A STEADY-STATE}

\section{A. An LMI-based condition}

Proceeding from Definition 1 let us rewrite (3), (4) in the compact form

$$
\bar{u}_{i}\left(g_{i}^{\top} \bar{u}+k_{i}\right)=-P_{i}, i \in \mathcal{M}
$$

where we have defined

$$
G^{\top}(0)=:\left[\begin{array}{llll}
g_{1} & g_{2} & \ldots & g_{m}
\end{array}\right] .
$$

It is clear then that the system admits an equilibrium if and only if, for the given values of $g_{i}, k_{i}$ and $P_{i}$, the quadratic equations (8) admit a solution (in $\bar{u}$ ).

The analysis of solvability of this kind of equations is the subject of study of classical quadratic mapping theory (see [8] and references therein). A direct application of Lemma 1 given in Appendix A yields the following result.

Proposition 1: Assume there exists a diagonal matrix $T:=\operatorname{diag}\left\{t_{i}\right\} \in \mathbb{R}^{m \times m}$ such that

$$
\left[\begin{array}{cc}
T G(0)+G^{\top}(0) T & T k \\
(T k)^{\top} & 2 \underline{1}_{m}^{\top} T P
\end{array}\right]>0 .
$$

Then, there is no constant steady-state for the system (1), (2).

The necessary condition of Proposition 1 is formulated in terms of LMIs - hence powerful convex optimization tools [2], [5] can be exploited to check it.
Let us compare it with (6) - recalling that (5) is always satisfied. Applying Schur's complement we have that (10), is equivalent to the inequalities

$$
\begin{gathered}
T G(0)+G^{\top}(0) T>0 \\
\underline{1}_{m}^{\top} T P>\frac{1}{2}(T k)^{\top}\left[T G(0)+G^{\top}(0) T\right]^{-1} T k .
\end{gathered}
$$

The second inequality states that if the weighted extracted power $\left(\sum_{i=1}^{m} t_{i} P_{i}\right)$ exceeds a lower bound then there is no equilibrium-provided the first inequality holds. On the other hand, condition (6) states that if there is an equilibrium the effective extracted power $\left(\sum_{i=1}^{m} P_{i}\right)$ should not exceed a certain upper bound. It is important to underscore that neither one of the conditions is sufficient for existence of equilibria. There are two facts that make the result of Proposition 1 more interesting. First, under some conditions discussed in the next section feasibility of the LMI is necessary and sufficient. Second the inclusion of free weighting factors $T$ gives a significant degree of freedom. Moreover, the search of the desired $T$ (if it exists) can be performed in a numerically efficient way. These facts are clearly illustrated in the example of Subsection VI-B.

\section{B. An alternative necessary condition}

Taking $T>0$ as a particular case of Proposition 1 yields an alternative necessary condition, which admits a very simple proof given in Appendix B-via completion of squares as done in [10].

Proposition 2: Assume there exists a positive definite diagonal matrix $T:=\operatorname{diag}\left\{t_{i}\right\} \in \mathbb{R}^{m \times m}$ such that (11) and

$$
\underline{1}_{m}^{\top} T P>\frac{1}{2}(T k)^{\top}\left[T G(0)+G^{\top}(0) T\right]^{-1} T k
$$

hold. Then, there is no constant steady-state for the system (1), (2).

Clearly, when $T=I_{m}$ conditions (11), (13) agree with (5) and (6), respectively, providing then an extension to the result in [10].

\section{ON SUFFICIENCY OF THE LMI CONDITION}

Proposition 1 provides a necessary condition, that is, if there exists constant steady-state, then LMI (10) has no solutions. The following question regarding sufficiency of this statement arises naturally.

Q3. Is it true that the lack of solutions of (10) implies solvability of equations (8)?

\section{A. On the role of convexity}

Question Q3 is closely related to convexity properties of images for quadratic transformations, see [8]. Indeed, the key point in the proof of Lemma 1 was the separation of the point $-P$ and the image of the mapping $f(\bar{u})$-denoted $\mathcal{F}$ and defined in (32). If a set is convex and closed, the lack of a strictly separating hyperplane is necessary and sufficient condition for a point to be feasible. Thus we arrive to the following complement to Proposition 1. 
Proposition 3: If the set $\mathcal{F}$ is convex - that is the case if $m=1$ or $m=2$ and (11) is solvable-equations (8) have a solution if and only if the LMI (10) is not feasible.

There are numerous results on convexity of quadratic images [3], [8]. For instance, as indicated in the proposition, $m \leq 2$ implies convexity. Unfortunately, for $m>2$ the set $\mathcal{F}$ is usually non-convex. In [9] a test to check convexity/nonconvexity of $\mathcal{F}$ is given. Thus, for a particular example one can examine sufficiency of the LMI condition. More precisely, if non-convexity is identified, there exists a $P \in \mathbb{R}^{m}$ such that (8) has no solution, and equations (11) have no solution either. However, it is hard to give the answer for a particular $P$.

\section{B. An illustrative example}

The next example illustrates two interesting aspects of the problem discussed in this section.

A1. It shows that lack of solutions of the LMI (10) does not imply solvability of equations (8) - providing a negative answer to question Q3.

A2. It is clear from the necessary condition (6) that for sufficiently large values of the extracted powers $P_{i}$ the system does not admit a steady-state solution. It looks natural to suggest that if equations (8) have a solution, there still will be a solution for smaller $P_{i}$. The example shows that this conjecture is not true in general.

Consider the case $m=3$ and

$G(0)=\left[\begin{array}{ccc}1 & -\frac{1}{2} & 1 \\ -\frac{1}{2} & 1 & -1 \\ -2 \epsilon & -2 \epsilon & 1\end{array}\right], k=\left[\begin{array}{c}-\frac{3}{2} \\ -\frac{1}{2} \\ -1\end{array}\right], P=\left[\begin{array}{c}\frac{1}{2} \\ \frac{1}{2} \\ \frac{1}{4}\end{array}\right]$,

where $\epsilon$ is a small number. Define

$$
f_{i}(\bar{u}):=\bar{u}_{i}\left(g_{i}^{\top} \bar{u}+k_{i}\right), i \in \mathcal{M} .
$$

Some simple calculations show that

$$
\begin{aligned}
f_{1}(\bar{u}) & =\bar{u}_{1}^{2}-\frac{1}{2} \bar{u}_{1} \bar{u}_{2}+\bar{u}_{1} \bar{u}_{3}-\frac{3}{2} \bar{u}_{1} \\
f_{2}(\bar{u}) & =\bar{u}_{2}^{2}-\frac{1}{2} \bar{u}_{1} \bar{u}_{2}-\bar{u}_{2} \bar{u}_{3}-\frac{1}{2} \bar{u}_{2} \\
f_{3}(\bar{u}) & =\bar{u}_{3}^{2}-2 \epsilon \bar{u}_{3}\left(\bar{u}_{1}+\bar{u}_{2}\right)-\bar{u}_{3} .
\end{aligned}
$$

To establish A1 we notice that the inequalities

$$
f_{i}(\bar{u})+P_{i}>0, i \in \mathcal{M},
$$

have a solution $\bar{u}=\left(1,1, \frac{1}{2}\right)$, which is checked by direct substitution. On the other hand, the system

$$
f_{i}(\bar{u})=-P_{i}, i \in \mathcal{M},
$$

has no solution. Recalling Proposition 3 this proves the nonconvexity of the set $\mathcal{F}$. $^{2}$

We proved above that the system has no equilibrium with power $P_{3}=\frac{1}{4}$. However, if this power is increased to $P_{3}=$ $\frac{1}{4}+2 \epsilon$ it has equilibria, contradicting the conjecture of $\mathrm{A} 2$ above. These two facts underscore the complicated topology of the solution set of the quadratic equations in question.

\footnotetext{
${ }^{2}$ The latter can also be established invoking the results of [9].
}

\section{Analysis of Stability of a Given Equilibrium POINT}

In this section we assume the system (1), (2) has a steadystate and analyze the stability —in the sense of Lyapunov-of the associated equilibrium point of its state-space realization. Therefore, a state description of the system is required. That is,

$$
\begin{aligned}
\dot{x} & =A x+B u \\
y & =C x+D u+k \\
y_{i} u_{i} & =-P_{i}, \quad i \in \mathcal{M},
\end{aligned}
$$

where $x$ is the state vector of dimension equal to the McMillan degree ${ }^{3}$ of $G(s)$ and $A, B, C, D$ are constant matrices, of suitable dimensions, such that

$$
G(s)=C(s I-A)^{-1} B+D .
$$

In Section III it has been shown that existence of a steadystate of the system (1), (2) is equivalent to existence of a constant vector $\bar{u}$ solution of (8). It is clear that, given $\bar{u}$, the associated equilibrium point of (16) is

$$
\bar{x}=-A^{-1} B \bar{u},
$$

with condition (5) ensuring that $A$ is full rank.

To streamline the presentation of our result define the parameterized matrices

$$
\begin{aligned}
R(\bar{u}) & :=\operatorname{diag}\left\{g_{i}^{\top} \bar{u}+k_{i}\right\}+\operatorname{diag}\left\{\bar{u}_{i}\right\} D \in \mathbb{R}^{m \times m} \\
S(\bar{u}) & :=\operatorname{diag}\left\{\bar{u}_{i}\right\} C \in \mathbb{R}^{m \times n} \\
M(\bar{u}) & :=A-B R^{-1}(\bar{u}) S(\bar{u}) \in \mathbb{R}^{n \times n},
\end{aligned}
$$

with the vectors $g_{i} \in \mathbb{R}^{m}$ the columns of $G^{\top}(0)$ as defined in (9).

Proposition 4: Assume the system (1), (2) admits a steady-state with associated constant vector $\bar{u}$ solution of (8).

R1. The equilibrium point (17) of the system state-space realization (16) is locally asymptotically stable if

$$
\mathcal{R}_{e}\left\{\lambda_{i}[M(\bar{u})]\right\}<0, i \in \mathcal{M},
$$

where $\lambda_{i}[\cdot]$ denotes the eigenvalues and $M(\bar{u})$ is defined in (18).

$\mathrm{R} 2$. The equilibrium is unstable if there exists $i \in \mathcal{M}$ such that

$$
\mathcal{R}_{e}\left\{\lambda_{i}[M(\bar{u})]\right\}>0 .
$$

Proof: The proof is a straightforward application of Lyapunov's First Method to the system (16). First, we notice that the system matrix for the first order approximation of the system (16) around the equilibrium point $\bar{x}$ is

$$
A+\left.B \frac{\partial u}{\partial x}\right|_{x=\bar{x}, u=\bar{u}}
$$

\footnotetext{
${ }^{3}$ That is, the dimension of a minimal realization of $G(s)$.
} 
So, the remaining task is to compute the partial derivative. Now, defining the rows of the matrices $C$ and $D$ as

$$
C=:\left[\begin{array}{c}
c_{1}^{\top} \\
c_{2}^{\top} \\
\vdots \\
c_{m}^{\top}
\end{array}\right], D=:\left[\begin{array}{c}
d_{1}^{\top} \\
d_{2}^{\top} \\
\vdots \\
d_{m}^{\top}
\end{array}\right],
$$

we can write the (implicit) control equation $y_{i} u_{i}=-P_{i}$ as

$$
u_{i}\left(c_{i}^{\top} x+d_{i}^{\top} u+k_{i}\right)=-P_{i}, i \in \mathcal{M} .
$$

Differentiating (20) with respect to $x$ we get

$$
\begin{aligned}
{\left[\operatorname{diag}\left\{c_{i}^{\top} x+d_{i}^{\top} u+k_{i}\right\}+\right.} & \left.\operatorname{diag}\left\{u_{i}\right\} D\right] \frac{\partial u}{\partial x} \\
& +\operatorname{diag}\left\{u_{i}\right\} C=0, \quad i \in \mathcal{M}
\end{aligned}
$$

Evaluating the identity above at the point $(\bar{x}, \bar{u})$ and using (17) and the definition of $S(\bar{u})$ we get

$$
\begin{aligned}
{\left[\operatorname{diag}\left\{\left(-c_{i}^{\top} A^{-1} B+d_{i}^{\top}\right) \bar{u}+k_{i}\right\}+\right.} & \left.\operatorname{diag}\left\{\bar{u}_{i}\right\} D\right] \frac{\partial u}{\partial x} \\
& =-S(\bar{u}), \quad i \in \mathcal{M}
\end{aligned}
$$

The proof is completed using the fact that

$$
G(0)=D-C A^{-1} B
$$

solving for the partial derivative to get

$$
\frac{\partial u}{\partial x}=-R^{-1}(\bar{u}) S(\bar{u})
$$

and replacing in (19).

\section{Two Illustrative EXAMPLES}

\section{A. A single port RLC circuit}

The linear RLC circuit with constant voltage source shown in Fig. 1 has been used in studies with CPLs in [1], [7], [11]. The transfer function $G(s)$ is given by

$$
G(s)=\frac{L s+r}{L C s^{2}+\left(r \mathrm{C}+\frac{L}{r_{c}}\right) s+\frac{r}{r_{c}}+1},
$$

with

$$
k=\frac{E}{1+\frac{r}{r_{c}}} .
$$

Notice that

$$
G(0)=\frac{r}{\frac{r}{r_{c}}+1}=: g
$$

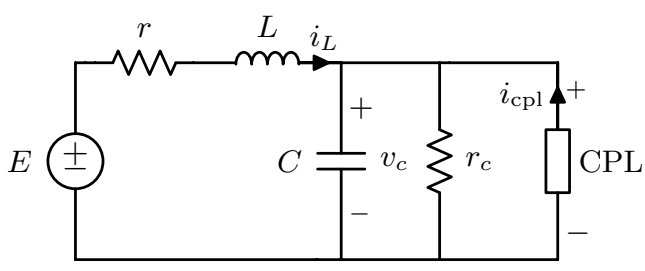

Fig. 1. Linear RLC circuit with a CPL.
Since $m=1$ the condition (6) is necessary and sufficient for the existence of a steady-state and it takes the form

$$
P \leq \frac{k^{2}}{4 g}=\frac{E^{2}}{4 r\left(\frac{r}{r_{c}}+1\right)}
$$

Assuming that (21) is satisfied we will invoke now Proposition 4 to study the stability of the equilibria. Defining the state vector

$$
x:=\left[\begin{array}{c}
i_{L} \\
v_{c}
\end{array}\right]-\frac{E}{1+\frac{r}{r_{c}}}\left[\begin{array}{c}
\frac{1}{r_{c}} \\
1
\end{array}\right],
$$

it is easy to see that the system admits a state representation of the form (16) with $(u, y)=\left(i_{c p l}, v_{c}\right)$ and

$$
A:=-\left[\begin{array}{cc}
\frac{r}{L} & \frac{1}{L} \\
-\frac{1}{\mathrm{C}} & \frac{1}{\mathrm{C} r_{c}}
\end{array}\right], B:=\left[\begin{array}{l}
0 \\
\frac{1}{\mathrm{C}}
\end{array}\right], C^{\top}:=\left[\begin{array}{l}
0 \\
1
\end{array}\right],
$$

Replacing the system data in (18) yields

$$
M(\bar{u})=\left[\begin{array}{cc}
-\frac{r}{L} & -\frac{1}{L} \\
\frac{1}{\mathrm{C}} & -\frac{1}{\mathrm{C}}\left[\frac{1}{r_{c}}+q(\bar{u})\right]
\end{array}\right],
$$

where we defined the function

$$
q(\bar{u}):=\frac{\bar{u}}{g \bar{u}+k} .
$$

Now, we compute $\bar{u}$ from (8), which takes the form

$$
g \bar{u}^{2}+k \bar{u}+P=0 .
$$

Since all coefficients of the quadratic equation are positive both roots are real negative. Moreover, the term $g \bar{u}+k$ is positive and, consequently, $q(\bar{u})<0$.

The characteristic polynomial of the matrix $M(\bar{u})$ is given as

$$
\begin{aligned}
\operatorname{det}[s I-M(\bar{u})]=s^{2}+\left[\frac{r}{L}+\right. & \left.\frac{1}{\mathrm{C}}\left(\frac{1}{r_{c}}+q(\bar{u})\right)\right] s \\
& \left.+\frac{1}{L \mathrm{C}}\left[1+\frac{r}{r_{c}}+r q(\bar{u})\right)\right]
\end{aligned}
$$

Some lengthy, but straightforward calculations, show that for the smallest root of (22) the polynomial above always has an unstable root. Hence, the associated equilibrium is unstable for all parameter values. On the other hand, for the greatest root of (22), the characteristic polynomial may be stable or unstable depending on the system parametersproperty that is inherited by the corresponding equilibrium point. The same situation holds true if there is just one equilibrium, that is, if $k^{2}=4 g P$.

\section{B. A multiport dc linear circuit}

Fig. 2 shows a de linear circuit with two CPLs. The transfer function matrix $G(s)$, with $\bar{y}=\operatorname{col}\left(v_{1}, v_{2}\right), \bar{u}=$ $\operatorname{col}\left(i_{\mathrm{cpl}_{1}}, i_{\mathrm{cpl}_{2}}\right)$ and $k=\operatorname{col}(E, E)$, is

$$
G(s)=\frac{1}{d(s)}\left[\begin{array}{ll}
n_{11}(s) & n_{12}(s) \\
n_{21}(s) & n_{22}(s)
\end{array}\right]
$$

where $n_{11}(s)=L_{1} C_{2} L_{2} s^{3}+\left(C_{2} L_{1} r_{2}+C_{2} L_{2} r_{1}\right) s^{2}+$ $\left(C_{2} r_{1} r_{2}+L_{1}\right) s+r_{1}, n_{12}(s)=n_{21}(s)=L_{1} s+r_{1}$, $n_{22}(s)=C_{1} L_{1} L_{2} s^{3}+\left(C_{1} L_{1} r_{2}+C_{1} L_{2} r_{1}\right) s^{2}+\left(C_{1} r_{1} r_{2}+\right.$ $\left.L_{1}+L_{2}\right) s+r_{1}+r_{2}$, and $d(s)=C_{1} C_{2} L_{1} L_{2} s^{4}+\left(C_{1} C_{2} L_{1} r_{2}+\right.$ 
$\left.C_{1} C_{2} L_{2} r_{1}\right) s^{3}+\left(C_{1} C_{2} r_{1} r_{2}+C_{1} L_{1}+C_{2} L_{1}+C_{2} L_{2}\right) s^{2}+$ $\left(C_{1} r_{1}+C_{2} r_{1}+C_{2} r_{2}\right) s+1$.

Then,

$$
G(0)=\left[\begin{array}{cc}
r_{1} & r_{1} \\
r_{1} & r_{1}+r_{2}
\end{array}\right] .
$$

Using Proposition 1 results in the LMI condition

$$
\left[\begin{array}{ccc}
2 t_{1} r_{1} & \left(t_{1}+t_{2}\right) r_{1} & t_{1} E \\
\left(t_{1}+t_{2}\right) r_{1} & 2 t_{2}\left(r_{1}+r_{2}\right) & t_{2} E \\
t_{1} E & t_{2} E & 2\left(t_{1} P_{1}+t_{2} P_{2}\right)
\end{array}\right]>0 \text {. }
$$

Since we are dealing with a two port system non-feasibility of the LMI is necessary and sufficient for existence of equilibria as indicated in Proposition 3.

TABLE I

PARAMETERS FOR THE CIRCUIT IN FIG. 2

\begin{tabular}{c|c|c|c}
\hline \hline$r_{1}=0.04 \Omega$ & $L_{1}=78.0 \mu \mathrm{H}$ & $C_{1}=2.0 \mathrm{mF}$ & $E=24.0 \mathrm{~V}$ \\
\hline$r_{2}=0.06 \Omega$ & $L_{2}=98.0 \mu \mathrm{H}$ & $C_{2}=1.0 \mathrm{mF}$ & \\
\hline \hline
\end{tabular}

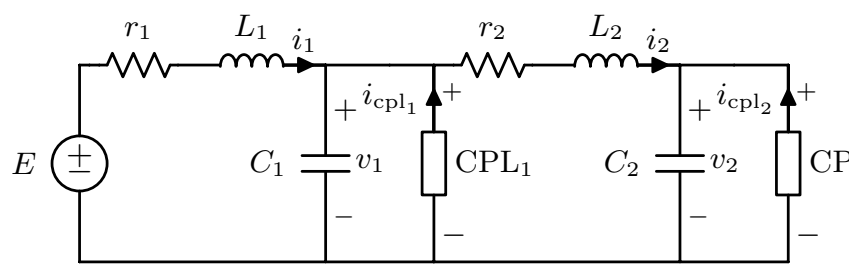

Fig. 2. Linear dc circuit with two CPLs.

Fig. 3 shows the evaluation using a gridding approach ${ }^{4}$ of the LMI (10) on the $P_{2}$ vs $P_{1}$ plane with the circuit parameters in Table I. In the blue region the LMI (23) is feasible and, then, no equilibria exist for the example. Conversely, in the green region the LMI (23) is not feasible and, then, an equilibrium for the system exists. The red curve in the plane is the numerically obtained boundary for the existence of equilibria that, as indicated in Proposition 3, coincides with the boundary predicted by Proposition 1 .

In the figure we also show in yellow the straight line

$$
P_{2}+P_{1}=\frac{1}{2} k^{\top}\left[G(0)+G^{\top}(0)\right]^{-1} k=3600
$$

which corresponds to the upper bound on the extracted power for existence of equilibria obtained from the inequality in (6). As seen from the figure the bound is, indeed, necessary but far from being sufficient.

\section{The CASE of Port-Hamiltonian Systems}

In [10] the question of existence of equilibria of controlled $\mathrm{pH}$ systems with constant dissipation connected to CPLs is also studied. The dynamics of these systems is given by

$$
\begin{aligned}
\dot{x} & =[J(d)-R] \nabla H(x)+k+g(x) u \\
y & =g^{\top}(x) \nabla H(x)
\end{aligned}
$$

${ }^{4} \mathrm{CVX}$, a package for specifying and solving convex programs, has been used to solve the semidefinite programming feasibility problem [5].

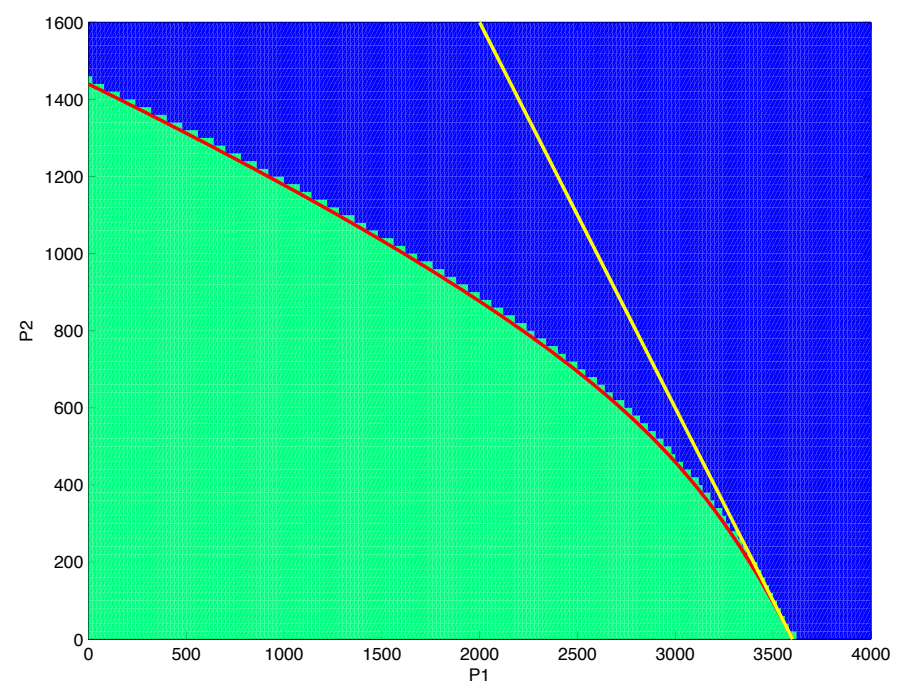

Fig. 3. Boundaries for existence of equilibria in the plane of extracted powers $P_{2}$ vs $P_{1}$ (in $\mathrm{W}$ ).

where $\nabla=\left(\frac{\partial}{\partial x}\right)^{\top}, x \in \mathbb{R}^{n}$ is the state vector, $d \in \mathbb{R}^{q}$ is a control signal, $H: \mathbb{R}^{n} \rightarrow \mathbb{R}$ is the system energy function, $k \in \mathbb{R}^{n}$ are constant external sources, the vectors $u, y \in \mathbb{R}^{m}$ are the port variables connected-through the input matrix $g: \mathbb{R}^{n} \rightarrow \mathbb{R}^{n \times m}$ - to CPLs, i.e., verifying (2). The interconnection matrix $J: \mathbb{R}^{q} \rightarrow \mathbb{R}^{n \times n}$ is of the form

$$
J(d)=J_{0}+\sum_{i=1}^{q} J_{i} d_{i}
$$

where the constant matrices $J_{i} \in \mathbb{R}^{n \times n}$ satisfy the skewsymmetry condition

$$
J_{i}=-J_{i}^{\top}, i=0,1, \ldots, q .
$$

The dissipation matrix is constant and verifies $R=R^{\top}>0$.

As discussed in [10] our motivation to consider this class of systems is that they suitably describe the dynamic behavior of power converters, under the assumption of a sufficiently fast switching frequency, with $d$ representing the duty cycle [4], [6].

Evaluating the time derivative of the total energy along the trajectories of (24), and using (2) and (25), yields the power balance equation of the $\mathrm{pH}$ system

$$
\begin{aligned}
\dot{H} & =-(\nabla H)^{\top} \dot{x} \\
& =-(\nabla H)^{\top}\{[J(d)-R] \nabla H(x)+k+g(x) u\} \\
& =-(\nabla H)^{\top} R \nabla H(x)+k+y^{\top} u \\
& =-(\nabla H)^{\top} R \nabla H+(\nabla H)^{\top} k-\mathbf{1}_{m}^{\top} P,
\end{aligned}
$$

where we clearly identify the dissipated, supplied and extracted power terms. It is clear that a necessary condition for existence of an equilibrium of (24) is that $\dot{H}=0$, which in its turn is equivalent to solvability of the quadratic equation

$$
0=-v^{\top} R v+v^{\top} k-\mathbf{1}_{m}^{\top} P,
$$

for some constant vector $v \in \mathbb{R}^{n}$, that corresponds to

$$
v:=\nabla H(\bar{x}),
$$


with $\bar{x} \in \mathbb{R}^{n}$ the associated equilibrium. Notice that the quadratic equation (27) are of the same form of equations (36), with $T=I_{m}$, analysed in Proposition 2 .

Now, to translate the condition of existence of constant equilibrium of the co-energy variables $\nabla H(x)$ to the energy variables ${ }^{5} x$ we need to assume - as done in [10]—-that the mapping $\nabla H: \mathbb{R}^{n} \rightarrow \mathbb{R}^{n}$ is surjective.

We have the proposition below, whose proof follows as a corollary of Proposition 2 taking $R=G(0)$ and $T=I_{m}$.

Proposition 5: The $\mathrm{pH}$ system (24) with Hamiltonian function such that $\nabla H(x)$ is surjective admits an equilibrium $\bar{x} \in \mathbb{R}^{n}$ only if

$$
\mathbf{1}_{m}^{\top} P \leq \frac{1}{4} k^{\top} R^{-1} k
$$

In [10] it is claimed that the condition above is sufficient when $n-q=1$. This claim, which was a consequence of the incorrect definition of a steady state indicated in Section II, is unfortunately wrong.

Similarly to the case discussed in Section II there is a clear physical interpretation of condition (28), with $\frac{1}{4} k^{\top} R^{-1} k$ being an upper bound on the power dissipated in steadystate, that should exceed the extracted constant power $\mathbf{1}_{m}^{\top} P$ to ensure the existence of equilibria.

\section{CONCLUSIONS}

We have studied the problems of derivation of conditions for existence of a steady-state for multi-port, LTI systems with CPLs and analysis of the stability of the associated equilibrium points. The main contributions of the paper are the following

C1. Prove that for single-port systems the simple test for existence of equilibria (6), given in [10], is necessary and sufficient, while for multi-port systems is only necessary.

C2. An extension to the necessary condition of [10] given in terms of an LMI has been derived.

C3. It has been show that the LMI condition is also sufficient if the set $\mathcal{F}$ is convex, which is the case for $m \leq 2$.

C4. Assuming the steady-state exists, a simple eigenvalue test has been given to verify the stability (or instability) of the associated equilibrium.

C5. In Section VII the sufficient version of the results is extended to the case when the LTI circuit is replaced by the class of perturbed port-Hamiltonian systems considered in Section IV of [10], which contains the important case of switched power converters.

The results can be directly extended to circuits where voltage (or current) sources appear also in the state equations.

\footnotetext{
${ }^{5}$ We recall that in $\mathrm{pH}$ modeling of electrical systems $\nabla H(x)$ corresponds to the co-energy variables, i.e. inductor currents and capacitor voltages while $x$ are the energy variables inductor fluxes and capacitor charges.
}

That is, systems of the form

$$
\begin{aligned}
\dot{x} & =A x+B u+w \\
y & =C x+D u+\kappa \\
y_{i} u_{i} & =-P_{i}, i \in \mathcal{M},
\end{aligned}
$$

with $w \in \mathbb{R}^{n}$ and $\kappa \in \mathbb{R}^{m}$ constant vectors. The results given above apply verbatim simply defining the new constant vector

$$
k:=\kappa-C A^{-1} w .
$$

\section{REFERENCES}

[1] M. Belkhayat, R. Cooley and A. Witulski, Large signal stability criteria for distributed systems with constant power loads, Proc. of the 26th Annual IEEE Power Electronics Specialists Conference (PESC'95), pp. 1333-1338, 1995.

[2] S. Boyd and L. Vanderberghe, Convex optimization, Cambridge University Press, 2004.

[3] A. Dymarsky, On the Convexity of Image of a Multidimensional Quadratic Map, arXiv:1410.2254, 2014.

[4] G. Escobar, A. J. van der Schaft and R. Ortega, A Hamiltonian viewpoint in the modeling of switching power converters, Automatica, Vol. 35, no. 189, pp. 445-452, 1999.

[5] M. Grant and S. Boyd, CVX: Matlab Software for Disciplined Convex Programming (version 2.1), http://cvxr.com/cvx, 2014.

[6] M. Hernandez-Gomez, R. Ortega, F. Lamnabhi-Lagarrigue, and G. Escobar. Adaptive PI stabilization of switched power converters. IEEE Transactions on Control Systems Technology, 18(3):688-698, 2009.

[7] H. Mosskull, Optimal stabilization of constant power loads with input LC-filters, Control Engineering Practice, Vol. 27, pp. 61-73, 2014.

[8] B. Polyak, Convexity of quadratic transformations and its use in control and optimization, Journal of Optimization Theory and Applications, 99, 553-583, 1998.

[9] B. Polyak and E. Gryazina, Convexity/nonconvexity certificate for power flow analysis, ISESO-2015, Heidelberg, 2015

[10] S. Sanchez, R. Ortega, R. Griñó, G. Bergna and M. Molinas-Cabrera, Conditions for existence of equilibrium points of systems with constant power loads, IEEE Transactions on Circuits and Systems I, Vol. 61, No. 7, pp. 2204-2211, 2014.

[11] S.D. Sudhoff, S.F. Glover, P.T. Lamm, D.H. Schmucker and D.E. Delisle, Admittance space stability analysis of power electronic systems, IEEE Transactions on Aerospace and Electronic Systems, Vol. 36, No. 3, pp. 965-973, 2000.

\section{Appendix A: Proof of Proposition 1}

The following lemma is instrumental in the proof of Proposition 1.

Lemma 1: Consider $m$ quadratic functions of the form $f_{i}$ : $\mathbb{R}^{m} \rightarrow \mathbb{R}$

$$
f_{i}(\bar{u}):=\frac{1}{2} \bar{u}^{\top} \mathcal{A}_{i} \bar{u}+\bar{u}^{\top} \mathcal{B}_{i}, i \in \mathcal{M}
$$

where $\mathcal{A}_{i}=\mathcal{A}_{i}^{\top} \in \mathbb{R}^{m \times m}$ and $\mathcal{B}_{i} \in \mathbb{R}^{m}$. For $T:=$ $\operatorname{diag}\left\{t_{i}\right\} \in \mathbb{R}^{m \times m}$ define

$$
\mathcal{A}(T):=\sum_{i=1}^{m} t_{i} \mathcal{A}_{i}, \mathcal{B}(T):=\sum_{i=1}^{m} t_{i} \mathcal{B}_{i}, \mathcal{P}(T):=\sum_{i=1}^{m} t_{i} P_{i}
$$

If the LMI in $T$

$$
\left[\begin{array}{cc}
\mathcal{A}(T) & \mathcal{B}(T) \\
\mathcal{B}^{\top}(T) & 2 \mathcal{P}(T)
\end{array}\right]>0
$$

is feasible, then equations

$$
f_{i}(\bar{u})=-P_{i}, i \in \mathcal{M}
$$

have no solution. 
Proof: Write the equations (31) in vector form as $f(\bar{u})=-P$. Define the set

$$
\mathcal{F}:=\left\{f(\bar{u}): \bar{u} \in \mathbb{R}^{m}\right\},
$$

that is the image of $\mathbb{R}^{m}$ under the quadratic map $f: \mathbb{R}^{m} \rightarrow$ $\mathbb{R}^{m}$. Let us minimize a linear function $\sum_{i=1}^{m} t_{i} z_{i}$ on $\mathcal{F}$ provided that $\mathcal{A}(T)>0$ :

$\alpha:=\min _{z \in \mathcal{F}} \sum_{i=1}^{m} t_{i} z_{i}=\min _{\bar{u}} \sum_{i=1}^{m} t_{i} f_{i}(\bar{u})=-\frac{1}{2} \mathcal{B}(T)^{\top} \mathcal{A}(T)^{-1} \mathcal{B}(T)$.

On the other hand, using the definition of $\mathcal{P}(T)$, and if

$$
-\mathcal{P}(T)<\min _{z \in \mathcal{F}} \sum_{i=1}^{m} t_{i} z_{i}
$$

it means that the hyperplane

$$
\sum_{i=1}^{m} t_{i} z_{i}=\frac{1}{2}[\alpha-\mathcal{P}(T)]
$$

strictly separates $-P$ and $\mathcal{F}$, hence equations (31) have no solution. On the other hand, the inequalities

$$
\mathcal{A}(T)>0, \quad \mathcal{P}(T)>\frac{1}{2} \mathcal{B}(T)^{\top} \mathcal{A}(T)^{-1} \mathcal{B}(T)
$$

are equivalent to (30) due to lemma on Schur complement (see e.g., [2], Appendix A.5.5).

To obtain Proposition 1 from Lemma 1 we define

$$
\begin{aligned}
\mathcal{A}_{i} & :=e_{i} e_{i}^{\top} G(0)+G^{\top}(0) e_{i} e_{i}^{\top} \\
\mathcal{B}_{i} & :=k_{i} e_{i}
\end{aligned}
$$

with $e_{i} \in \mathbb{R}^{m}$ the $i$-th Euclidean basis vector. Whence, the terms in Lemma 1 can be written as

$$
\mathcal{A}(T)=T G(0)+G^{\top}(0) T, \mathcal{B}(T)=T k, \mathcal{P}(T)=\underline{1}_{m}^{\top} T P .
$$

\section{ApPENDIX B: PRoOF OF PROPOSITION 2}

To simplify the notation define the positive definite matrix

$$
\mathcal{T}:=\frac{1}{2}\left[T G(0)+G^{\top}(0) T\right]>0 .
$$

Condition (13) then becomes

$$
\frac{1}{4}(T k)^{\top} \mathcal{T}^{-1}(T k)-\underline{1}_{m}^{\top} T P<0 .
$$

Proceeding from (8), multiply the $i$-th equation by $t_{i}$ and sum them up to get

$$
\bar{u}^{\top} T G(0) \bar{u}+\bar{u}^{\top} T k=-\mathbf{1}_{m}^{\top} T P .
$$

Clearly, solvability of (8) implies solvability of (36). Now, extracting the symmetric part of the quadratic form and recalling the definition of $\mathcal{T}$ in (34) we have

$$
\bar{u}^{\top} T G(0) \bar{u}=\bar{u}^{\top} \mathcal{T} \bar{u} \text {. }
$$

Replacing the expression above and completing the square, it is easy to see that (36) is equivalent to

$$
\begin{aligned}
\left(\bar{u}+\frac{1}{2} \mathcal{T}^{-1} T k\right)^{\top} \mathcal{T}(\bar{u}+ & \left.\frac{1}{2} \mathcal{T}^{-1} T k\right) \\
& =\frac{1}{4}(T k)^{\top} \mathcal{T}^{-1}(T k)-\mathbf{1}_{m}^{\top} T P .
\end{aligned}
$$

Condition (34) ensures that the quadratic form in the lefthand side of the equation above is non-negative, while condition (35) makes the right-hand side negative, contradicting solvability of (36). This, in its turn, contradicts solvability of (8) and, consequently, (3), (4) admit no solution. 